\title{
Is it possible to prevent autism?
}

\section{Proceeding}

Throughout the whole life only of two generations autism declared itself as a real threat to the psychobiological health of the mankind. It is suggested that in several decades not only in the countries of the third world, but also in quite economically safe ones, the number of autists can make a half of the population and more. Therefore, already today we are forced not only to establish the fact of growing of the number of patients, but also to call the specialists of different profiles for searching of the ways of primary autism prevention. We see the first and main way in the improvement of the period of conceptionpregnancy-childbirth, consisting of several important components.

The first component: keeping to the healthy lifestyle by parents. Entering the reproductive age in the condition of psychophysical health is a banal well-known statement which, nevertheless, is often neglected, but only in Ancient Egyptian times the healthy lifestyle was admired and people spoke about it poetically and elevated, like about a living woman: "Her name - the Health. She is a daughter of Exercises, born from Temperance. Roses blossom on her cheeks; morning freshness breathes from her lips; the joy, harmonious with innocence and modesty shines in her eyes; her heart is full of fun; she sings, while walking ..." (the citation by Kalnish V. V., 2016). Perhaps, psychophysical health will also not prevent autism, but at least it will reduce the range of pathology, having reduced its manifestation to Asperger syndrome, which is simpler for the parents to reconcile to, and in many cases they can even be proud of the progress of their special child. But if it is about a need of having of the child by the family from the ASD (autism spectrum disorders) risk group, keeping to a healthy lifestyle, taking care of the health by parents is the first and the most important thing from all the other desirable conditions. Internal homeostasis sanitation of parents, and, first of all-mothers is especially important in this case.

The immunological homeostasis of mother fluctuates, and can be both complicating and after sanitation favorable for the birth of a healthy child. A. B. Poletaiev, A. A. Poletaieva and coauthors (2015) consider that one of the probable mechanisms, provoking ASD in many cases is the phenomenon of maternal immune imprinting, based on the transplacental flux of the maternal autoantibodies of the JgG class to the fetus and the epigenetic "setup" of the immune system of the future child. The features of the repertoires of maternal autoantibodies can depend on the prevalence in her these or those bacterial and virus associates, and also on specific features of the woman's organism. In fact being for the mother adaptive, for the future child they can be pathogenic and will form a basis for the emergence of ASD in him. Revealing of the latent virus infection carrier state is determined quite easily today. In particular, the virus of human papilloma under certain conditions disturbs fetus neurogenesis, and its removal before the planned pregnancy will not take a lot of time. I.e. sanitation of the mother with a risk of the birth of the child with ASD can prevent the action of this factor.

Also we find the instructions on possible pathological influence of the mother's homeostasis on the fetus in earlier works. In 1973 S.F. Semenov (S.F. Semenov, K. N. Nazarov, A. P. Chuprikov, 1973) paid attention that the pregnant mother, having endogenous and procedural psychosis due to her own neuroimmunological processes
Volume 9 Issue 4 - 2018

\author{
Chuprikov AP \\ Shupyk National Medical Academy of Postgraduate Education, \\ Ukraine
}

\begin{abstract}
Correspondence: Chuprikov AP, Chair of General, Child and Jurical Psychiatry and Narcology, Shupyk National Medical Academy of Postgraduate Education, Kiev, Ukraine, Email apchuprikov@gmail.com
\end{abstract}

Received: December 05, 2017 | Published: July 10, 2018

in the perinatal period can form autoimmune encephalopathy in the fetus. The last one, being an external factor in relation to the fetus genome, can sharply complicate the state of its health and aggravate the condition of its brain. Perhaps, the effect of complication of the clinical disease symptoms by transferring from a sick mother to her posterity consists in it. One more illustration of the possibility of transferring of the pathological factors from the mother to the fetus, leading to the emergence of the corresponding pathology in the last one, is the complex of works by G. A. Vartanian, B. I. Klementeva (1991), specifying the postural asymmetry factor (PAF). These works, in our opinion, are estimated in quite narrow bounds of cerebral spastic infantile paralysis etiopathogenesis though their value can also concern a great number of others lateralizated and non-lateralizated brain injuries of the fetus and the newborn, which in subsequent can be predictors of weakness of protective brain forces. Today the chemical and neurophysiological characteristics of PAF and even the fact that it can be found in amniotic liquid are known and, if to inject it to a young mouse, it is possible to provoke the brain injury of the known laterality. Thus, one more detail of possible pathology transferring (both obvious, and latent) from the mother to the fetus, about which in due time O.E. Viazov (1962) told as about "from one organ to another organ phenomenon", has been discovered, i.e. the mother's pathology can be transmitted to the fetus organ of the same name in non-genetic way.

Another statement, deserving attention, is the following: as a result of the laborious analysis of the state of health of 2000000 children of Sweden, who were born since 1982 to 2006, carried out by the international group of scientists, 14516 people with the different types of ASD have been revealed. It allowed discovering that at least $50 \%$ of the responsibility in the ASD emergence is connected with genetics, and the rest $50 \%$ with the adverse combination of the environmental factors. This research allowed answering a frequent question of the risk of the repeated birth of the child with ASD-it turned out that the presence of the brother or sister with ASD makes the risk of birth of the child with autism 10 higher in comparison with the allpopulation risk. In the presence of the cousin the risk also increases twice as much. I.e., at the individual level the risk of autism is directly proportional to the degree of genetic proximity with the relative-autist (Avi Reichenberg, Sven Sandin, according to Leliukhina M., 2015). 
The second component consists in determination of the genetic risk of the birth of a sick child. On this subject there are many publications, from which it follows that autism is connected with the sex of the child, and in boys it is observed 4-5 times more often. Is germ selection, according to the sex possible? Yes, in some Asian countries the parents, want to have a person, who can help them at their old age, and they consider the son is the best for this, but not the daughter make such operations. And now there is a million-big surplus of grooms and the deficiency of brides there. It is quite possible that in the families with the child-autist for the purpose of risk reduction of the birth of the sibs with a similar disease sometimes selection of germs, according to the sex can be carried out. The question of the genetic risk most sharply arises in the family, which already have probandautist and after the consultation with the geneticist about the degree of danger of the repeated case of autism in the family you should not fall into despair. The matter is that the doctors-practitioners know well: in the families, inclined to have pathological genes, both sick and healthy children are born. We assume that the signs of gene pathology can vary under the influence of the external environment factors, i.e. epigenetics can not only aggravate the disease course, but also can resist to it. For example, I happened to examine two couples of adult monogerminal twins 35 years ago. One of the twins in both couples had schizophrenia, and the other was almost healthy, i.e. these couples were discordant on schizophrenia. And this happens very seldom, as this disease concordance among monogerminal twins reaches $80-85 \%$. The explanation of this discordance was simple - the twins differed from each other in their handedness: one was a lefthander, the second one - the right-handed person. When I give lectures and ask doctors: who had schizophrenia, lefthanders or right-handed ones? For some reason most often I get the answer: lefthanders. I should correct the mistake: no, right-handed persons were sick. I.e. the later oosperm division into two germs, when several stages of oosperm divisions have passed, and laterality came out, i.e. the right and left parts can have a serious impact on the destiny of the person, having identical set of pathological genes with the sibs, he may not have the disease signs.

Consulting children-autists and asking about their handedness, I often noted that autism in left-handers, though it exists, but looks differently - it correlates with hyperactivity and psychopathological originality. Thus, the change of the lateral profile, its difference from the specific laterality characteristics, inherent in the population of this region, can limit manifestations of the pathological genes partially or completely. That the line item, stated above can be quite realistic we can find in the statement of Stephen H. Friend, the president of the biotechnological company Sage Bionetworks, studying almost healthy people with catastrophic genetic mutations, which might have provoked incurable illnesses in the childhood. "Instead of observation of the patients with the diagnosed diseases it is necessary to study the people, who were simply obliged to get sick, but by some chance avoided it" (Friend S., 2016). The third component: in case of the planned conception the early acquaintance of the family with the perinatal psychologist, having his psychotherapeutic work experience with a family, is necessary. Normalization of the intra family relations, reliefs of fears and alarm of the pregnant woman, her psychophysical sanitation, at last, training of the techniques of the pregnant woman's communication with the growing fetus-all this is possible within the activities of the perinatal psychologist. As supervisory we had to study the work of the perinatal psychologist G. V. Shpatakovskaia (Odessa) and see how, as a result of her work, the clients-women (and their husbands) enjoy the pregnancy and the expectations of the childbirth turn into joy and happiness of the meeting with a newborn. The process of the childbirth in these cases proceeded without complications.

Also in case of the planned conception choosing of the time for conception, taking into account local climatic and geographical conditions, is important. The dynamics of ups and downs of births of such children is given in the chapter, devoted to the seasonal prevalence of births of children-autists in Ukraine for the first time in the CIS countries. We hope that from now the specialists, consulting the parents can count a decrease in the risk of autism of the planed child in connection with a seasonality factor. For example, it is possible to plan conception-the birth of the new child with a delay of 3-6 months after that term, peculiar to the first child. A decrease in the risk of the autist's birth can be promoted by parents' consideration of the fact of reduction of the number of births of sick children in February-March with conception in May-June (in Ukraine). Choosing of the time for the healthy child's birth can also depend on another temporary factor. In the internet there was a statement of the American researchers that the birth of the second child during the first two years after the first one increases the risk of the birth of the second child-autist twice as much. The same thing is observed, if the birth of the second child occurs later, than in 6 years after the first one. Let's wait for verification of this statement by other scientists, but we can consider, it supplements the opinion that the risk of the birth of the child-autist from elderly fathers sharply increases (Martsenkovskyi I. A., 2013). The fourth component: mobilization of the obstetricians on prevention of the prenatal conflicts between the mother and the fetus. It is clear, that it is possible in case of the careful control on the condition of the pregnant woman and the fetus! For example, the restless behavior of the fetus, as the evidence of its possible hypoxemic condition, usually comes to the end with the child's birth with a psychoorganic defect and it is good if it is just limited to deficit of attention and hyperactivity. Naturally, it seriously complicates the clinical picture of autism and complicates both the medical and correctional psychology and pedagogical help.

The fifth component consists in a reasonable pharmacological background-folic acid, vitamins, etc. Pregnant women seem to know that taking of the agents, promoting normal formation of the nervous tube, then brain structures of the fetus, however, there are mothers, who either did not know or neglected this. The sixth component: "pregnant women have to look only on beautiful things around". This hackneyed phrase speaks about the century-old wisdom of the people, about every possible protection of pregnant women from the psychotraumatizing factors and crises of any origin. In the neuropsychological plan pregnant women are extraordinary vulnerable, sensitive to adverse effects. Our experience with neurologic and psychological disorders of pregnant women and parturient women convincingly testifies to it. Thus, the tolerance of the people around to the pregnant woman's whims, tolerance to her sometimes unusual behavior can promote the normal gestation course that will allow avoiding hard moments, even with genetic predisposition. In our clinic E.S. Galchin $(2015,2016)$ found out that the birth of the child with autism in the faraway areas is preceded by the following negative social and psychological factors: the low level of the material welfare in combination with the negative microclimate in the family (to $64,2 \%$ ), the growth of the perinatal pathology (in comparison with a control group): preeclampsia, early and late gestosis, threat of abortion, fetus infection. During the delivery experimental group mothers were observed to show statistically authentically raised weak birth traumas, pelvic presentation, etc., concerning the patrimonial activity in combination with fetus asphyxia, cord entanglement. We find similar 
data in the research of I. A. Martsenkovskii' (2013). It is possible to assume that adverse factors of the period of conception, pregnancy and delivery can promote implication of genetic troubles and even in a dose-dependent way aggravate its clinic.

One of the perspective directions of the psychophysical improvement of the mother or both parents, who already have one child-autist, is the complex kinesitherapy in combination with the elements of craniosacral therapy (T. V. Chernaia, 2014). The considerable number of mothers has psychogenic, somatoform disorders, which make them disabled and are a serious obstacle for the birth of the new posterity. Psychologist-kinesitherapist T.V. Chernaia carries out psychosensorial integration both for the child-autist, and his mother, restoring both health of the child, and the positive intra family atmosphere. An important role in the psychophysical improvement is played by communication with animals (dolphins, horses, dogs). At the same time bathing with dolphins can promote both conception (in case of sterility), and the mother's and fetus' health stabilization. Consulting of pregnant women in the dolphinarium of Ukraine and
Russia is carried out by obstetricians-gynecologists and perinatal psychologists. Dolphins are capable to distinguish the condition of pregnancy of the women and to show them special sensitivity and care. When making simple contact with animals it becomes clear that the dolphin can easily determine the gender of the future child (E. Igrunova's observations in "Nemo" dolphinarium, Kiev). After several sessions of swimming of the pregnant woman with dolphins the threat of gestation deterioration, abortions disappears, the birth proceeds without complications. Gradually the number of cases of the almost healthy children's births goes up. Based on the information stated above, we urge the experts and parents to take this hard way of fight against autism!

\section{Acknowledgements}

None.

\section{Conflict of interest}

The author declares that there is no conflict of interest. 